\title{
EL DESEMPEÑO LABORAL INFLUENCIA EN EL CUMPLIMIENTO DE LOS OBJETIVOS INSTITUCIONALES DE LA UNIVERSIDAD NACIONAL JOSÉ FAUSTINO SÁNCHEZ CARRIÓN
} THE LABOR PERFORMANCE INFLUENCE IN FULFILLING THE INSTITUTIONAL
OBJECTIVES OF THE UNJFSC

Héctor Serafín López Traslaviña ${ }^{1}$, Ronald Luis Ramos Pacheco ${ }^{2}$, César Alexander López Ramos ${ }^{2}$,

\section{RESUMEN}

En la investigación titulada: El Desempeño laboral influencia en el cumplimiento de los objetivos institucionales de la UNJFSC, tuvo como objetivo analizar el desempeño laboral y su influencia en los objetivos institucionales de la UNJFSC.

El tipo de investigación fue básica, explicativo, casuístico, su diseño no experimental, transversal de nivel descriptivo explicativo. La población estuvo constituida por 273 trabajadores administrativos nombrados, la muestra fue probabilística quedando un total de 160 trabajadores. Se aplicó un cuestionario validado y con un valor de confiabilidad alfa de cronbach de 0.86 .

Se evidencia que el desempeño laboral influye significativamente en los objetivos institucionales de la UNJFSC. Se concluye que a un nivel regular se presentó el desempeño laboral y los objetivos institucionales de los trabajadores administrativos nombrados en la Universidad Nacional José Faustino Sánchez Carrión.

Palabras clave: Desempeño Laboral; Objetivos Institucionales.

\begin{abstract}
In the research titled: The Labor performance influence in the fulfillment of the institutional objectives of the UNJFSC, it had as objective to analyze the labor performance and its influence in the institutional objectives of the UNJFSC.

The type of research was basic, explanatory, casuistic, its non-experimental design, transversal descriptive descriptive level. The population was constituted by 273 named administrative workers, the sample was probabilistic leaving a total of 160 workers. A validated questionnaire with a chronbach alpha reliability of 0.86 was applied.

It is evident that the labor performance significantly influences the institutional objectives of the UNJFSC. It is concluded that at a regular level the work performance and the institutional objectives of the administrative workers named at the National University José Faustino Sánchez Carrión were presented.
\end{abstract}

Key words: Labor Performance; Institutional Objectives.

Universidad Nacional José Faustino Sánchez Carrión de Lima (Lima - Perú). Email:vinvestigación@unjfsc.edu.pe 


\section{INTRODUCCIÓN}

La presente investigación titulada El Desempeño laboral influencia en el cumplimiento de los objetivos institucionales de la Universidad Nacional José Faustino Sánchez Carrión. La Universidad, se ha evidenciado que existe cierto grado de ineficacia en los procesos ejecutados por el personal administrativo, lo que indica que no se está gestionando adecuadamente su talento humano. Por lo tanto, esto podría convertirse en una barrera para contribuir con el cumplimiento de los objetivos institucionales de la calidad de la educación superior, según las exigencias establecidas en la Ley Universitaria $\mathrm{N}^{\mathrm{o}} 30220$ y sus organismos de reguladores como la SUNEDU quienes establecen control y supervisión de "trámites, permisos y requisitos gracias a los cuales las instituciones universitarias operan con licitud y legalidad, al amparo de una garantía pública sobre el alcance de sus actividades: autorización, reconocimiento, certificación, habilitación, títulos, lo que se conoce como calidad sustantiva de la universidad.

En la era de la información y el conocimiento en que las organizaciones públicas operan actualmente, la administración del talento humano se ha convertido en un pilar estratégico para la gestión, pues el personal constituye su capital intelectual cuya presencia y participación activa es tan importante como los procesos, la infraestructura y la misma tecnología. Sin embargo, a menudo en las organizaciones encontramos trabajadores inconformes con sus puestos de trabajo, desorientados acerca de la forma de ejecutar las tareas o simplemente desmotivados y sin compromiso hacia la institución esto, sin duda influye negativamente e imposibilita el desarrollo de la organización.

La falta de procesos especializados para organizar el personal tales como la orientación, diseño de puestos y evaluación del personal están provocando bajo nivel de desempeño, limitando que el personal administrativo de la UNFSC desarrolle su proactividad, creatividad y espíritu innovador, al no existir la suficiente orientación de las funciones y objetivos alcanzar, induce a que el personal direccione equivocadamente sus actividades y esfuerzos, por ende se da la confusión de tareas, además que la escaza división del trabajo incurre en la baja especialización del personal en sus tareas y como consecuencia se da el retraso de la entrega de documentación e información entre dependencias de la Institución.
Por lo antes mencionado es importante describir la problemática que se presenta en la Institución; así por ejemplo, no se utilizan herramientas desempeño laboral adecuada, lo que se hace, es dar cumplimiento a lo estipulado en la reglamentación interna general, puesto que los subprocesos de reclutamiento y selección son deficientes; como consecuencia se asignan profesionales con diferente perfil profesional al que se requiere para cubrir la vacante laboral, se desaprovecha el potencial humano y se limita al mismo a desarrollar nuevas ideas, experiencias y expectativas en el cumplimiento de sus tareas.

De acuerdo a lo anterior, se planteó la siguiente pregunta: ¿De qué manera el desempeño laboral influye en cumplimiento de los objetivos institucionales de la Universidad Nacional José Faustino Sánchez Carrión?

De acuerdo a los lineamientos de la Escuela de Posgrado de la Universidad Nacional José Faustino Sánchez Carrión, el presente trabajo de investigación, consta de VI Capítulos como son: El Capítulo I, Problema de investigación, comprende puntos esenciales para la investigación, integra: El planteamiento del problema, formulación del problema, justificación, limitaciones, antecedentes de estudio y objetivos; el Capítulo II corresponde al Marco Teórico, e incluye las bases teóricas relacionadas con las variables de estudio. Considera los siguientes temas: Desempeño laboral y su influencia en el cumplimiento de los objetivos institucionales, dentro del cual se analiza el concepto, estilos, teorías y evaluación, en el que analizamos minuciosamente el concepto, indicadores, procesos, teorías, evaluación, ventajas y dificultades, también se adjunta la definición de términos básicos; el Capítulo III trata sobre el Marco Metodológico, la metodología, trata y analiza todo el aspecto metodológico, tipos de estudio y diseño; asimismo, se señala población y muestra, método de investigación, técnicas e instrumentos de recolección de datos y métodos de análisis de datos, en el Capítulo IV, están los análisis de los resultados de la investigación y la contrastación de hipótesis, luego se presenta en el Capítulo $\mathrm{V}$ están la discusión de los resultados, en el capítulo VI están las conclusiones y recomendaciones, las conclusiones que como resultado de todo trabajo de investigación desplegado demuestra la prueba de hipótesis que en este caso resulta aceptada en una relación de carácter significativa. Igualmente se formulan las recomendaciones respectivas para superar la situación problemática determinado como resultado 
del trabajo de investigación, finalmente se presenta las referencias bibliografías y anexos. En los anexos se presentan la matriz de consistencia, cuestionario, los instrumentos y la base de datos.

\section{OBJETIVOS}

Objetivo General.

Analizar el desempeño laboral y su influencia en el cumplimiento de los objetivos institucionales de la Universidad Nacional José Faustino Sánchez Carrión.

\section{Objetivos Específicos.}

Explicar cómo influye la política de trabajo en los objetivos estratégicos de la Universidad Nacional José Faustino Sánchez Carrión.

Determinar la influencia de la gestión de rendimiento en los objetivos tácticos de la Universidad Nacional José Faustino Sánchez Carrión

La gestión de compensaciones influye en los objetivos operativos de la Universidad Nacional José Faustino Sánchez Carrión.

\section{MATERIALES Y METODOS}

El diseño de la investigación fue no experimental, porque nos permitió determinar las características del problema sin alterarlas o cambiarlas. El tipo de investigación a utilizarse según la finalidad es de tipo descriptiva, básica, aplicativa y casuística, debido a que se apoyara primeramente en el contexto teórico para luego establecerla correlación frente al problema.

Población. Está conformada por 273 trabajadores administrativos nombrados en la Universidad Nacional José Faustino Sánchez Carrión.

Muestra: Se ha utilizado el muestreo aleatorio simple porque cada elemento de la población tiene la misma posibilidad de ser seleccionado para integrar la muestra, ya que este principio permite obtener conclusiones en la muestra e inferir lo que pudiera ocurrir, a partir de ésta, en la población, con un elevado grado de pertinencia recomendado en investigaciones educacionales y estratificado porque se va a subdividir la población en subgrupos o estratos. Quedando como muestra 160 trabajadores.

Técnicas de recolección de datos

Entrevista.- permitió aplicar la encuesta y a la vez conoció el desempeño laboral y su influencia en los objetivos organizacionales y verificar la información obtenida a través del instrumento de investigación.

b) Encuesta.- Se tomó la información y se alcanzó los objetivos y se verifico las hipótesis.

c) Bibliográficas.- Que se constituyen en el soporte para el análisis de la investigación

Técnicas para el procesamiento de la información

a) Estadística descriptiva

Se describió los datos, los valores de dirección por variables utilizando la distribución de frecuencia y gráficos.

\section{RESULTADOS}

El desempeño laboral influye significativamente en los objetivos institucionales de la UNJFSC.

Tabla 1. Pruebas de Chi - Cuadrado de las variables desempeño laboral y objetivos institucionales

\begin{tabular}{llr}
\hline Descriptivo & Símbolo & \multicolumn{1}{c}{ Valor } \\
\hline Chi-Cuadrado & $\mathrm{X}^{2}$ & 37.57 \\
Grados de libertad & $\mathrm{Gl}$ & 4 \\
Significancia bilateral & $\mathrm{p}$-valor & 0 \\
Significancia estadística & alfa & 0.05 \\
Nivel de confianza & 1-alfa & $95 \%$ \\
Número de encuestados & $\mathrm{N}$ & 160 \\
\hline
\end{tabular}

Como el valor de significancia del estadístico es $\mathrm{p}=$ 0 ; menor que $\alpha=0.05$, entonces podemos rechazar la hipótesis nula y concluir que existe suficiente evidencia estadística para decir que el desempeño laboral influye significativamente en los objetivos institucionales de la Universidad Nacional José Faustino Sánchez Carrión.

La política de trabajo influye significativamente en los objetivos estratégicos de la UNJFSC.

Tabla 2. Prueba Chi - Cuadrado de las variables política de trabajo y objetivos estratégicos

\begin{tabular}{lcc}
\hline Descriptivo & Símbolo & Valor \\
\hline Chi-Cuadrado & $\mathrm{X}^{2}$ & 35.33 \\
Grados de libertad & $\mathrm{Gl}$ & 4 \\
Significancia bilateral & $\mathrm{p}$-valor & 0 \\
Significancia estadística & alfa & 0.05 \\
Nivel de confianza & 1-alfa & $95 \%$ \\
Número de encuestados & $\mathrm{N}$ & 160 \\
\hline
\end{tabular}


Interpretación:

Como el valor de significancia del estadístico es $\mathrm{p}=$ 0 ; menor que $\alpha=0.05$, entonces podemos rechazar la hipótesis nula y concluir que existe suficiente evidencia estadística para decir que la política de trabajo influye significativamente en los objetivos estratégicos de la Universidad Nacional José Faustino Sánchez Carrión.

La gestión de rendimiento influye significativamente en los objetivos tácticos de la UNJFSC.

Tabla 3. Prueba Chi-Cuadrado de las variables gestión de rendimiento y objetivos tácticos

\begin{tabular}{llr}
\hline Descriptivo & Símbolo & \multicolumn{1}{c}{ Valor } \\
\hline Chi - Cuadrado & $\mathrm{X}^{2}$ & 38.19 \\
Grados de libertad & $\mathrm{Gl}$ & 4 \\
Significancia bilateral & $\mathrm{p}$-valor & 0 \\
Significancia estadística & alfa & 0.05 \\
Nivel de confianza & 1-alfa & $95 \%$ \\
Número de encuestados & $\mathrm{N}$ & 160 \\
\hline
\end{tabular}

Interpretación:

Como el valor de significancia del estadístico es $\mathrm{p}=$ 0 ; menor que $\alpha=0.05$, entonces podemos rechazar lahipótesis nula y concluir que existe suficiente evidencia estadística para decir que la gestión de rendimiento influye significativamente en los objetivos tácticos de la Universidad Nacional José Faustino Sánchez Carrión.

La gestión de compensaciones influye significativamente en los objetivos operativos de la UNJFSC.

Tabla 4. Prueba Chi-Cuadrado de las variables gestión de compensaciones y objetivos operativos.

\begin{tabular}{llc}
\hline Descriptivo & Símbolo & Valor \\
\hline Chi - Cuadrado & $\mathrm{X}^{2}$ & 30.793 \\
Grados de libertad & $\mathrm{Gl}$ & 4 \\
Significancia bilateral & $\mathrm{p}$-valor & 0 \\
Significancia estadística & alfa & 0.05 \\
Nivel de confianza & 1-alfa & $95 \%$ \\
Número de encuestados & $\mathrm{N}$ & 160 \\
\hline
\end{tabular}

Interpretación:

Como el valor de significancia del estadístico es $\mathrm{p}=$ 0 ; menor que $\alpha=0.05$, entonces podemos rechazar la hipótesis nula y concluir que existe suficiente evidencia estadística para decir que la gestión de compensaciones influye significativamente en los objetivos operativos de la Universidad Nacional José Faustino Sánchez Carrión.

\section{DISCUSIÓN}

El resultado obtenido al contrastar la hipótesis general, mediante la prueba estadística Chi cuadrado dio el valor de 37.57 a un nivel confianza del 0,05, nos permite evidenciar estadísticamente que el desempeño laboral influye significativamente en los objetivos institucionales de la Universidad Nacional José Faustino Sánchez Carrión. Estos resultados son coincidentes con la investigación presentada por Quispe (2015) demuestra que el Clima organizacional se relaciona con el desempeño laboral en la Municipalidad Distrital de Pacucha, Andahuaylas, llego a la conclusión que los trabajadores de la municipalidad logran algunas veces realizar un regular desempeño. Otra investigación presentada por Huamani (2015) señala que el clima organizacional influye en el desempeño laboral de los conductores del tren del área de transporte del metro de Lima, la línea 1. En la investigación presentada por Arias (2001, citado por Fernando, J. y Romeo, U. (sf)) señalan que el desempeño laboral se refiere a la capacidad mental y psicomotriz necesaria para efectuar un trabajo o ejercer una ocupación. Mientras que Castro (2012) enfatiza en el clima organizacional determina en parte el comportamiento de los trabajadores, comportamiento que genera productividad en la organización a través de un desempeño laboral eficiente o deficiente según sea el caso.

En consecuente a la investigación se logró el primer objetivo específico 1 el cual consistió en explicar cómo influye la política de trabajo en los objetivos estratégicos de la Universidad Nacional José Faustino Sánchez Carrión. Al contrastar la hipótesis especifica 1, se observó que el valor del estadístico Chi cuadrado resultante fue de 35.34 y $\mathrm{p}=0$, resultado que nos indicó que el valor de significancia bilateral es menor que 0,05 por lo tanto se ha llegado a comprobar que la política de trabajo influye significativamente en los objetivos estratégicos de la Universidad Nacional José Faustino Sánchez Carrión. Este resultado es coincide con la investigación presentado por Latorre (2011) afirma que las organizaciones se adaptan al entorno mediante las estrategias y políticas empresariales la gestión de la fuerza 
laboral es clave a la hora de adaptarse a la demanda del entorno y esto puede llevar en muchas ocasiones al éxito o al fracaso de la empresa.

De la misma manera, para lograr el objetivo específico 2, consistió en determinar la influenciade la gestión de rendimiento en los objetivos tácitos de la Universidad Nacional José Faustino Sánchez Carrión. Los resultados de contrastar la hipótesis específica 2 condujeron a conjeturar que el valor del estadístico Chi cuadrado resultante fue de 38.19 y $\mathrm{p}=0$, resultado que indicó que el valor de significancia bilateral es menor que 0,05 por lo tanto se ha llegado a comprobar que la gestión de rendimiento influye significativamente en los objetivos tácitos de la Universidad Nacional José Faustino Sánchez Carrión. Estos resultados son similares al estudio realizado por los investigadores Villa y Velázquez (2009) quienes analizan el rendimiento de los trabajadores a través de la evaluación del desempeño, así mismo esta evaluación podría caracterizarse como un medio de aprovechamiento o explicación máxima del potencial y rendimiento de los trabajadores. Por otra parte en la investigación realizada por Arias (2001, citado por Fernando, J. y Romeo, U. (sf)) explica que la mayoría de los empleados demuestran interés por obtener los conocimientos necesarios de su puesto de trabajo, poseen aspectos conceptuales practico y tienen conocimiento de los procesos administrativos.

Así mismo, para lograr el objetivo específico 3, consistió en analizar de qué modo influye la gestión de compensaciones en los objetivos operativos de la Universidad Nacional José Faustino Sánchez Carrión. Los resultados de contrastar la hipótesis específica 3 condujeron a determinar que el valor del estadístico Chi cuadrado resultante fue de 30.79 y $\mathrm{p}=0$, resultado que nos indicó que el valor de significancia bilateral es menor que 0,05 por lo tanto se ha llegado a la conclusión que la gestión de compensaciones influye significativamente en los objetivos operativos de la Universidad Nacional José Faustino Sánchez Carrión. Estos resultados son concordantes con la investigación presentada por Arias (2001, citado por Fernando, J. y Romeo, U. (sf)) quien sostiene que la empresa le otorgue al trabajador oportunidades de ascenso por méritos en la organización, así como son reconocidos los logros obtenidos en el desempeño de las labores de los empleados, por otra parte los investigadores Zeithaml y Bitner (2012) señalan que la colaboración con los clientes y y de prestar el servicio con prioridad, destaca la atención y la prontitud con la que se hace frente a las solicitudes, preguntas, los reclamos y los problemas de los clientes.

\section{CONCLUSIONES}

Después del análisis realizado en la contrastación de la hipótesis general, se observó que el valor de la Chi cuadrado resultante fue de $37.57 \mathrm{y} p=0$, lo que indica que el valor de la significancia bilateral es menor que alfa $=0,05$. Por lo tanto se rechaza la hipótesis nula $(\mathrm{H} 0)$ y se acepta la hipótesis alternativa (H1), por lo que se concluye que el desempeño laboral influye significativamente en los objetivos institucionales de la Universidad Nacional José Faustino Sánchez Carrión.

Producto del análisis realizado en la contrastación de la hipótesis específica 1 , se observó que el valor de la Chi cuadrado resultante fue de 35.34 y p=0, resultado que indica que el valor de la significancia bilateral es menor que alfa $=0,05$. Por lo tanto se rechaza la hipótesis nula (H0) y se acepta la hipótesis alternativa (H1), por lo que se concluye que la política de trabajo influye significativamente en los objetivos estratégicos de la Universidad Nacional José Faustino Sánchez Carrión.

Según el valor encontrado en la contrastación de la hipótesis específica 2 , se observó que el valor la Chi cuadrado resultante fue de 38.19 y $\mathrm{p}=0$, resultado que nos indica que el valor de significancia bilateral es menor que alfa $=0,05$. Por lo tanto, se rechaza la hipótesis nula (H0) y se acepta la hipótesis alternativa (H1), por lo que se concluye que la gestión del rendimiento influye significativamente en los objetivos tácitos de la Universidad Nacional José Faustino Sánchez Carrión.

Según el valor encontrado en la contrastación de la hipótesis específica 3, se observó que el valor de la Chi cuadrado resultante fue de 30.79 y $p=0$, resultado que nos indica que el valor de significancia bilateral es menor que alfa $=0,05$. Por lo tanto, se rechaza la hipótesis nula $(\mathrm{H} 0)$ y se acepta la hipótesis alternativa (H1), por lo que se concluye que la gestión de compensaciones influye significativamente en los objetivos operativos de la Universidad Nacional José Faustino Sánchez Carrión. 


\section{REFERENCIA BIBLIOGRÁFICA}

Figueroa, A., \& Moyano, E. (2009). Tesis: Factores Laborales de Equilibrio entre Trabajo y Familia: Para Mejorar la Calidad de Vida Instituto de Estudios Humanísticos. Universum, 23.

Briones, G. (2002). Metodologa de la Investigación Cuantitativa en las Ciencias Sociales. (A. editores, Ed.) Bogota, Colombia.
Hernandez, R., Fernandez, C., \& Baptista, M. (2010). Metodologia de la Investigación (Quinta ed.). (S. D. Interamarica Editores, Ed.) Mexico: Mc GraW Hill.

Rodriguez, D. (2010). Gestion organiozacional (2004 ed., Vol. I). Santiago, Chile: Ediciones universidad catolicadechile.

Universidad Nacional José Faustino Sanchez Carrión.
(29 de Diciembre de 2012). Cuadro de asignación de personal - CAP. Huacho, Peru.

Autoridad Nacional del Servicio civil. (02 de Junio de 2015). SERVIR. Recuperado el 02 de Junio de 2015, de http://www.servir.gob.pe/le yserviciocivil/ 